\title{
Convection in Couple-Stress Magneto-Fluid
}

\author{
Gursharn Jit Singh ${ }^{1}$, Pardeep Kumar ${ }^{2}$ \\ ${ }^{1}$ Department of Mathematics, SCD Govt. College Ludhiana (Pb), India. \\ ${ }^{2}$ Department of Mathematics, ICDEOL, Himachal Pradesh University, Summer-Hill, Shimla-171005,
}

\begin{abstract}
Double-diffusive convection in a couple-stress fluid in the presence of uniform vertical magnetic field through porous medium using linearized stability theory and normal mode analysis is studied. For the case of stationary convection, the stable solute gradient, magnetic field and couple-stress are found to have stabilizing effects whereas medium permeability has destabilizing effect on the system. The dispersion relation is also analyzed analytically. Further, it is found that the solute gradient and uniform magnetic field introduce oscillatory modes in the system, which was non-existent in their absence. The sufficient conditions for the nonexistence of overstability are also obtained.
\end{abstract}

Keywords: couple-stress fluid, double-diffusive convection, linearized stability theory, porous medium, uniform vertical magnetic field,

\section{Introduction}

The derivation of the basic equations of a layer of fluid heated from below in porous medium, using Boussinesq approximation, has been given by Joseph [1976]. The study of a layer of fluid heated from below in porous media is motivated both theoretically and by its practical applications in engineering disciplines. Among the applications in engineering disciplines one can find the food process industry, chemical process industry, solidification and centrifugal casting of metals. The development of geothermal power resources has increased general interest in the properties of convection in porous medium. Lapwood [1948] has studied the stability of convective flow in a porous medium using Rayleigh's procedure. The Rayleigh instability of a thermal boundary layer in flow through a porous medium has been considered by Wooding [1960]. When the fluid slowly percolates through the pores of the rock, the gross effect is represented by the well-known Darcy's law. An extensive and updated account of convection in porous media has been given by Nield and Bejan [1999].

A detailed account of the theoretical and experimental results of the onset of thermal instability (Bénard convection) in a fluid layer under varying assumptions of hydrodynamics and hydromagnetics has been given in the celebrated monograph by Chandrasekhar [1981]. Veronis [1965] has investigated the problem of thermohaline convection in a layer of fluid heated from below and subjected to a stable salinity gradient. The buoyancy forces can arise not only from density differences due to variations in temperature but also from those due to variations in solute concentration. Thermosolutal convection problems arise in oceanography, limnology and engineering. The investigation of thermosolutal convection is motivated by its interesting complexities as a double diffusion phenomena as well as its direct relevance to geophysics and astrophysics. Stomell et al. [1956] did the pioneering work regarding the investigation of thermosolutal convection. This work was elaborated in different physical situations by Stern [1960] and Nield [1967]. Examples of particular interest are provided by ponds built to trap solar heat (Tabor and Matz [1965]) and some Antarctic lakes (Shirtcliffe [1964]). The physics is quite similar in the stellar case in that Helium acts like salt in raising the density and in diffusing more slowly than heat. The conditions under which convective motion in double-diffusive convection are important (e.g. in lower parts of the Earth's atmosphere, astrophysics and several geophysical situation) are usually far removed from the consideration of a single component fluid and rigid boundaries and therefore it is desirable to consider a fluid acted on by a solute gradient and free boundaries.

The theory of couple-stress fluid has been formulated by Stokes [1966]. One of the applications of couple-stress fluid is its use to the study of the mechanisms of lubrications of synovial joints, which has become the object of scientific research. A human joint is a dynamically loaded bearing which has articular cartilage as the bearing and synovial fluid as the lubricant. When a fluid is generated, squeeze-film action is capable of providing considerable protection to the cartilage surface. The shoulder, ankle, knee and hip joints are the loaded-bearing synovial joints of the human body and these joints have a low friction coefficient and negligible wear. Normal synovial fluid is a viscous, non-Newtonian fluid and is generally clear or yellowish. According to the theory of Stokes [1966], couple-stresses appear in noticeable magnitudes in fluids with very large molecules.

Many of the flow problems in fluids with couple-stresses, discussed by Stokes, indicate some possible experiments, which could be used for determining the material constants, and the results are found to differ from those of Newtonian fluid. Couple-stresses are found to appear in noticeable magnitudes in polymer solutions for 
force and couple-stresses. This theory is developed in an effort to examine the simplest generalization of the classical theory, which would allow polar effects. The constitutive equations proposed by Stokes [1966] are:

$$
\begin{aligned}
& T_{(i j)}=\left(-p+\lambda D_{k k}\right) \delta_{i j}+2 \mu D_{i j}, \\
& T_{[i j]}=-2 \eta \vec{W}_{i j . k k}-\frac{\rho}{2} \vec{\varepsilon}_{i j s} G_{s},
\end{aligned}
$$

and

$$
M_{i j}=4 \eta \vec{\omega}_{j, i}+4 \eta^{\prime} \vec{\omega}_{i, j}
$$

where

$$
D_{i j}=\frac{1}{2}\left(V_{i, j}+V_{j, i}\right), \vec{W}_{i j}=-\frac{1}{2}\left(V_{i, j}-V_{j, i}\right)
$$

and

$$
\vec{\omega}_{i}=\frac{1}{2} \vec{\varepsilon}_{i j k} V_{k, j}
$$

Here $T_{i j}, T_{(i j)}, T_{[i j]}, M_{i j}, D_{i j}, \vec{W}_{i, j}, \vec{\omega}_{i}, G_{s}, \vec{\varepsilon}_{i j k}, \mathrm{~V}, \rho$ and $\lambda, \mu, \eta, \eta^{\prime}$, are stress tensor, symmetric part of $T_{i j}$, anti-symmetric part of $T_{i j}$, the couple-stress tensor, deformation tensor, the vorticity tensor, the vorticity vector, body couple, the alternating unit tensor, velocity field, the density and material constants respectively. The dimensions of $\lambda$ and $\mu$ are those of viscosity whereas the dimensions of $\eta$ and $\eta^{\prime}$ are those of momentum.

Since the long chain hyaluronic acid molecules are found as additives in synovial fluids, Walicki and Walicka [1999] modeled the synovial fluid as a couple-stress fluid. The synovial fluid is the natural lubricant of joints of the vertebrates. The detailed description of the joint lubrication has very important practical implications. Practically all diseases of joints are caused by or connected with a malfunction of the lubrication. The efficiency of the physiological joint lubrication is caused by several mechanisms. The synovial fluid is, due to its content of the hyaluronic acid, a fluid of high viscosity, near to a gel. Goel et al. [1999] have studied the hydromagnetic stability of an unbounded couple-stress binary fluid mixture under rotation with vertical temperature and concentration gradients. Sharma et al. [2002] have considered a couple-stress fluid with suspended particles heated from below. They have found that for stationary convection, couple-stress has a stabilizing effect whereas suspended particles have a destabilizing effect. In another study, Sunil et al. [2002] have considered a couple stress fluid heated from below in a porous medium in the presence of a magnetic field and rotation. Kumar et al [2004] have considered the thermal instability of a layer of a couple-stress fluid acted on by a uniform rotation, and have found that for stationary convection, the rotation has a stabilizing effect whereas couple-stress has both stabilizing and destabilizing effects.

Keeping in mind the importance in geophysics, soil sciences, ground water hydrology, astrophysics and various applications mentioned above, the double-diffusive convection in couple-stress fluid in the presence of uniform magnetic field through porous medium has been considered in the present paper.

\section{Formulation of the problem and perturbation equations}

Here we consider a layer of electrically conducting couple-stress fluid of thickness $d$ in porous medium heated and soluted from below so that the temperatures and solute concentrations at the bottom surface $\mathrm{z}=0$ are $T_{o}$ and $C_{o}$ and at the upper surface $\mathrm{z}=\mathrm{d}$ are $T_{1}$ and $C_{1}$ respectively, z- axis being taken as vertical. A uniform vertical magnetic field $\vec{H}(0,0, H)$ pervades the system.

Let $\delta \rho, \delta p, \theta, \gamma, \vec{q}(u, v, w)$ and $\vec{h}\left(h_{x}, h_{y}, h_{z}\right)$ denote the perturbations in density $\rho$, pressure p, temperature $\mathrm{T}$, solute concentration $\mathrm{C}$, velocity $(0,0,0)$ and magnetic field $\vec{H}(0,0, H)$ respectively. Then the linearized hydromagnetic equations relevant to the problem are

$$
\begin{aligned}
& \frac{1}{\varepsilon} \frac{\partial \vec{q}}{\partial t}=-\frac{1}{\rho_{0}} \nabla \delta p-\vec{g}\left(\alpha \theta-\alpha^{\prime} \gamma\right)-\frac{1}{k_{1}}\left(v-\frac{\mu^{\prime}}{\rho_{0}} \nabla^{2}\right) \vec{q}+\frac{\mu_{e}}{4 \pi \rho_{0}}(\nabla \times \vec{h}) \times \vec{H}, \\
& \nabla \cdot \vec{q}=0, \\
& E \frac{\partial \theta}{\partial t}=\beta w+\kappa \nabla^{2} \theta, \\
& E \frac{\partial \gamma}{\partial t}=\beta^{\prime} w+\kappa \nabla^{2} \gamma, \\
& \nabla \cdot \vec{h}=0,
\end{aligned}
$$


$\varepsilon \frac{\partial \vec{h}}{\partial t}=(\vec{H} \cdot \nabla) \vec{q}+\varepsilon \eta \nabla^{2} \vec{h}$,

where $E=\varepsilon+(1-\varepsilon) \frac{\rho_{s} c_{s}}{\rho c}$.

Here $\varepsilon$ is the medium porosity, $k_{1}$ is the medium permeability, and $\vec{g}(0,0,-g)$ is gravitational acceleration respectively. $k_{1}$ has the dimension of length squared. $\rho$, c and $\rho_{s}, c_{s}$ stand for density and specific heat of fluid and solid ( porous matrix) material respectively.

$v$ is the kinematic viscosity, $\mu^{\prime}$ is the couple-stress viscosity, $\kappa$ is the thermal diffusivity, $\alpha$ is the coefficient of thermal expansion, $\eta$ is the electrical resistivity ( all assumed to be constants), and $\mu_{e}$ stands for magnetic permeability.

The equation of state is

$$
\rho=\rho_{0}\left[1-\alpha\left(T-T_{0}\right)+\alpha^{\prime}\left(C-C_{0}\right)\right],
$$

where the suffix zero refers to values at the reference level $\mathrm{z}=0$ and so the change in density $\delta \rho$ caused by the perturbations $\theta$ and $\gamma$ in temperature and concentration, is given by

$$
\delta \rho=-\rho_{0}\left(\alpha \theta-\alpha^{\prime} \gamma\right) \text {. }
$$

The equation of state (7) contains a thermal coefficient of expansion $\alpha$ and an analogous solvent coefficient $\alpha^{\prime} . E^{\prime}$ is a solute parameter analogous to $\mathrm{E}$.

The steady state solution is

$$
\begin{aligned}
& \vec{q}=(0,0,0), T=T_{0}-\beta z, C=C_{0}-\beta z, \\
& \rho=\rho_{0}\left(1+\alpha \beta z-\alpha^{\prime} \beta^{\prime} z\right),
\end{aligned}
$$

where $\beta=\frac{T_{0}-T_{1}}{d}$ and $\beta^{\prime}=\frac{C_{0}-C_{1}}{d}$ are the magnitudes of uniform temperature and concentration gradients and are both positive as the temperature and concentration decrease upwards.

Now we consider the case in which both the boundaries are free, the medium adjoining the fluid is a perfect electrical conductor and temperature, concentration at a boundary are kept fixed. Then the boundary conditions appropriate to the problem are

$$
w=\frac{\partial^{2} w}{\partial z^{2}}=\theta=\gamma=\frac{\partial h_{z}}{\partial z}=0 \text { at } \mathrm{z}=0 \text { and } \mathrm{z}=\mathrm{d} .
$$

Equations (1)-(6) give

$$
\begin{aligned}
& {\left[\frac{1}{\varepsilon} \frac{\partial}{\partial t}+\frac{1}{k_{1}}\left(v-\frac{\mu^{\prime}}{\rho_{0}} \nabla^{2}\right)\right] \nabla^{2} w-g\left(\frac{\partial^{2}}{\partial x^{2}}+\frac{\partial^{2}}{\partial y^{2}}\right)\left(\alpha \theta-\alpha^{\prime} \gamma\right)-\frac{\mu_{e} H}{4 \pi \rho_{0}} \frac{\partial}{\partial z} \nabla^{2} h_{z}=0,} \\
& \left(E \frac{\partial}{\partial t}-\kappa \nabla^{2}\right) \theta=\beta w, \\
& \left(E \frac{\partial}{\partial t}-\kappa \nabla^{2}\right) \gamma=\beta^{\prime} w, \\
& \varepsilon\left(\frac{\partial}{\partial t}-\eta \nabla^{2}\right) h_{z}=H \frac{\partial w}{\partial z} .
\end{aligned}
$$

\section{Dispersion relation}

form

Analyzing the disturbances into normal modes, we assume that the perturbation quantities are of the

$$
\left[w, \theta, \gamma, h_{z}\right]=[W(z), \Theta(z), \Gamma(z), K(z)] \exp \left(i k_{x} x+i k_{y} y+n t\right),
$$

where $k_{x}, k_{y}$ are the wave numbers along the $x$ - and $y$-directions respectively, $k\left(=\sqrt{k_{x}^{2}+k_{y}^{2}}\right)$ is the resultant wave number and $\mathrm{n}$ is the growth rate which is, in general, a complex constant.

Using expression (13), equations (9)-(12) in non-dimensional form become 


$$
\begin{aligned}
& {\left[\frac{\sigma}{\varepsilon}+\frac{1}{P_{l}}-\frac{F}{P_{l}}\left(D^{2}-a^{2}\right)\right]\left(D^{2}-a^{2}\right) W+\frac{g d^{2} a^{2}}{v}\left(\alpha \Theta-\alpha^{\prime} \Gamma\right)-\frac{\mu_{e} H d}{4 \pi \rho_{0} v}\left(D^{2}-a^{2}\right) D K=0,} \\
& \left(D^{2}-a^{2}-E p_{1} \sigma\right) \Theta=-\left(\frac{\beta d^{2}}{\kappa}\right) W, \\
& \left(D^{2}-a^{2}-E^{\prime} q \sigma\right) \Gamma=-\left(\frac{\beta^{\prime} d^{2}}{\kappa^{\prime}}\right) W, \\
& \left(D^{2}-a^{2}-p_{2} \sigma\right) K=-\left(\frac{H d}{\varepsilon \eta}\right) D W,
\end{aligned}
$$

where we have put

$$
a=k d, \sigma=\frac{n d^{2}}{v}, \frac{x}{d}=x^{*}, \frac{y}{d}=y^{*}, \frac{z}{d}=z^{*} \text { and } D=\frac{d}{d z^{*}} .
$$

Here

$p_{1}=\frac{v}{\kappa}$ is the $\operatorname{Pr}$ andtl number,

$p_{2}=\frac{v}{\eta}$ is the magnetic $\operatorname{Pr}$ andtl number,

$q=\frac{v}{\kappa^{\prime}}$ is the Schmidt number,

$P_{l}=\frac{k_{1}}{d^{2}}$ is the dimensionless medium permeability

and

$F=\frac{\mu^{\prime}}{\rho_{0} d^{2} v}$ is the dimensionless couple - stress parameter.

We shall suppress the star $(*)$ for convenience hereafter.

Eliminating $\Theta, \Gamma$ and $\mathrm{K}$ between equations (14)-(17) and using the proper solution $W=W_{0} \sin \pi z, W_{0}$ being constant, we obtain the dispersion relation

$$
\begin{gathered}
R_{1}=\frac{(1+x)\left(1+x+E p_{1} \frac{\sigma}{\pi^{2}}\right)\left[\left\{\frac{\sigma_{1}}{\varepsilon \pi^{2}}+\frac{1}{P}+\frac{\pi^{2} F}{P}(1+x)\right\}\right]}{x}+S_{1} \frac{\left(1+x+E p_{1} \frac{\sigma}{\pi^{2}}\right)}{\left(1+x+E^{\prime} q \frac{\sigma}{\pi^{2}}\right)} \\
+\frac{Q_{1}}{\varepsilon} \frac{(1+x)\left(1+x+E p_{1} \frac{\sigma}{\pi^{2}}\right)}{x\left(1+x+p_{2} \frac{\sigma}{\pi^{2}}\right)},
\end{gathered}
$$

where

$$
R_{1}=\frac{g \alpha \beta d^{4}}{v \kappa \pi^{4}}, S_{1}=\frac{g \alpha^{\prime} \beta^{\prime} d^{4}}{v \kappa^{\prime} \pi^{4}}, Q_{1}=\frac{\mu_{e} H^{2} d^{2}}{4 \pi \rho_{0} v \eta \pi^{2}}, P=\pi^{2} P_{l}, \quad x=\frac{a^{2}}{\pi^{2}} .
$$

\section{The stationary convection}

For the stationary convection $\sigma=0$ and equation (18) reduces to

$$
R_{1}=\frac{(1+x)}{x}\left[\frac{(1+x)}{P}(1+\pi^{2} F \overbrace{1+x}^{-})+\frac{Q_{1}}{\varepsilon}\right]+S_{1} .
$$

Equation (19) yields

$\frac{d R_{1}}{d S_{1}}=+1$, 
$\frac{d R_{1}}{d P}=-\frac{(1+x)^{2}}{x P^{2}}\left(1+\pi^{2} F \widetilde{1+x}\right)$,

$\frac{d R_{1}}{d Q_{1}}=\frac{(1+x)}{\varepsilon x}$,

and

$\frac{d R_{1}}{d F}=\frac{\pi^{2}(1+x)^{3}}{x P}$,

which imply that couple-stress, stable solute gradient and magnetic field have stabilizing effects whereas medium permeability has destabilizing effect on the system.

The dispersion relation (19) is also analyzed numerically for various values of $S_{1}, P, Q_{1}$ and $F$. It is also evident from Fig. 1 - 4 that couple-stress, stable solute gradient and magnetic field have stabilizing effects whereas medium permeability has destabilizing effect on the system.

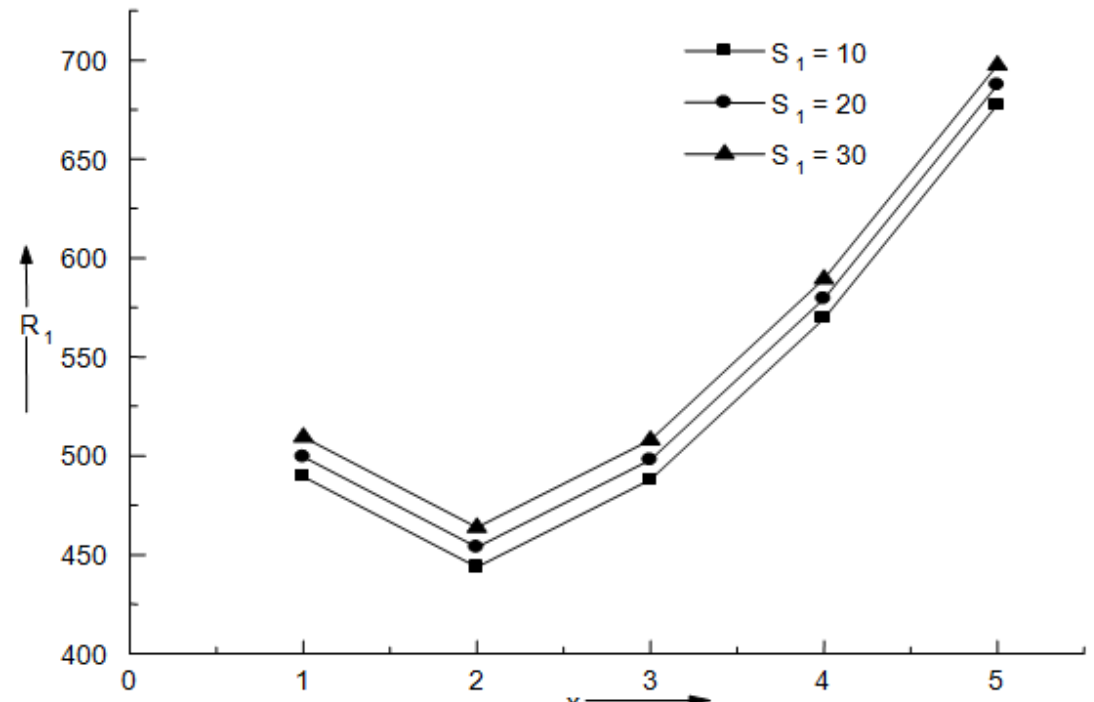

Figure 1: The variation of Rayleigh number $\left(R_{1}\right)$ with wave number $(X)$ for $P=10, F=10$.

$Q_{1}=100, \varepsilon=0.5$ and $S_{1}=10,20,30$.

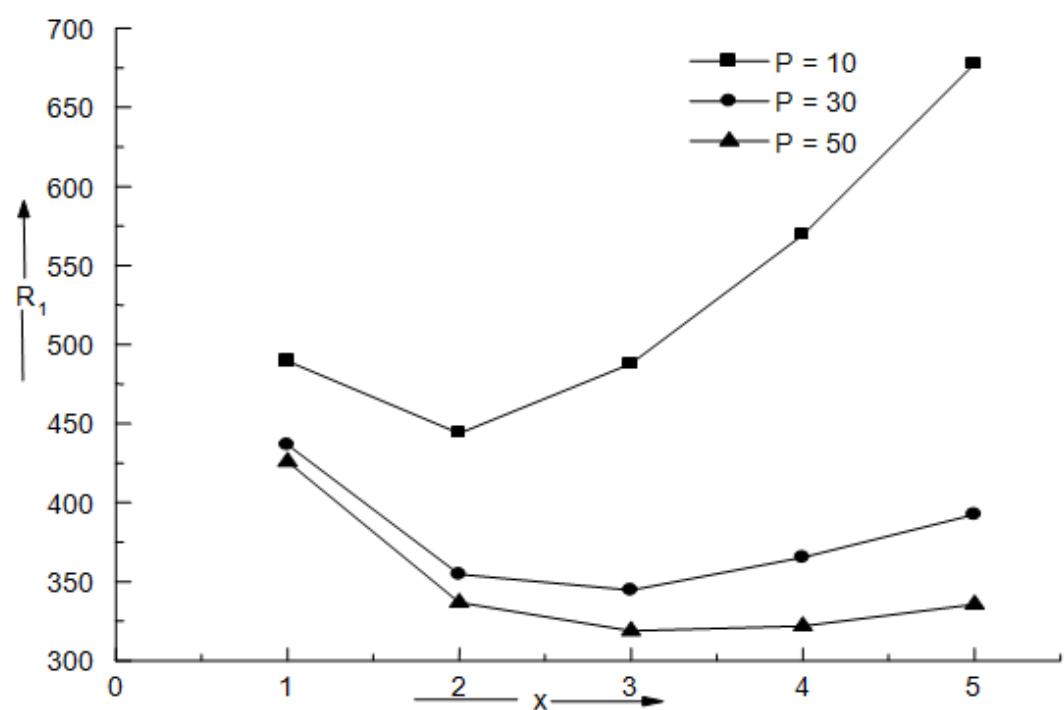

Figure.2: The variation of Rayleigh number $\left(R_{1}\right)$ with wave number $(X)$ for $F=10, Q_{1}=100$,

$S_{1}=10, \varepsilon=0.5$ and $P=10,30,50$ 


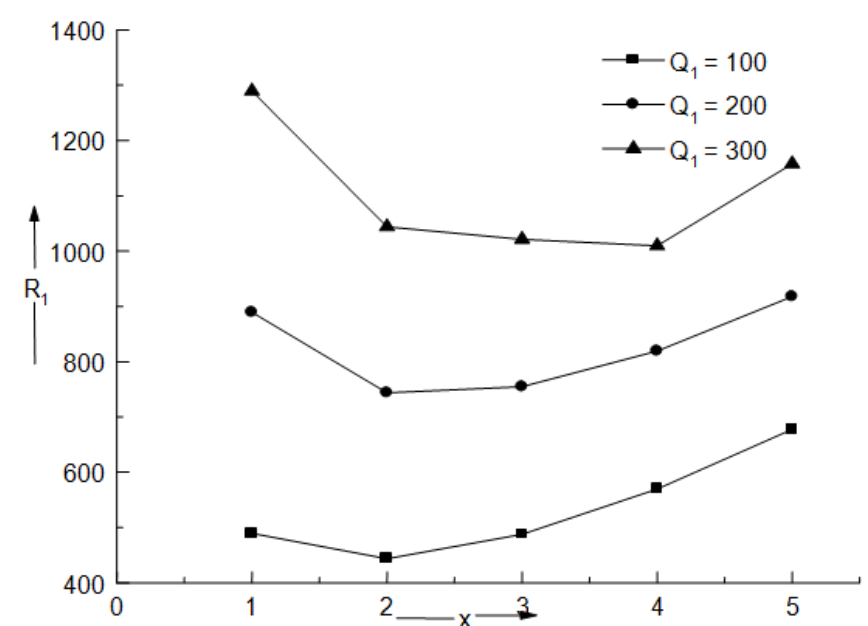

Figure 3: The variation of Rayleigh number $\left(R_{1}\right)$ with wave number $(x)$ for $P=10, F=10$,

$S_{1}=30, \varepsilon=0.5$ and $Q_{1}=100,200,300$.

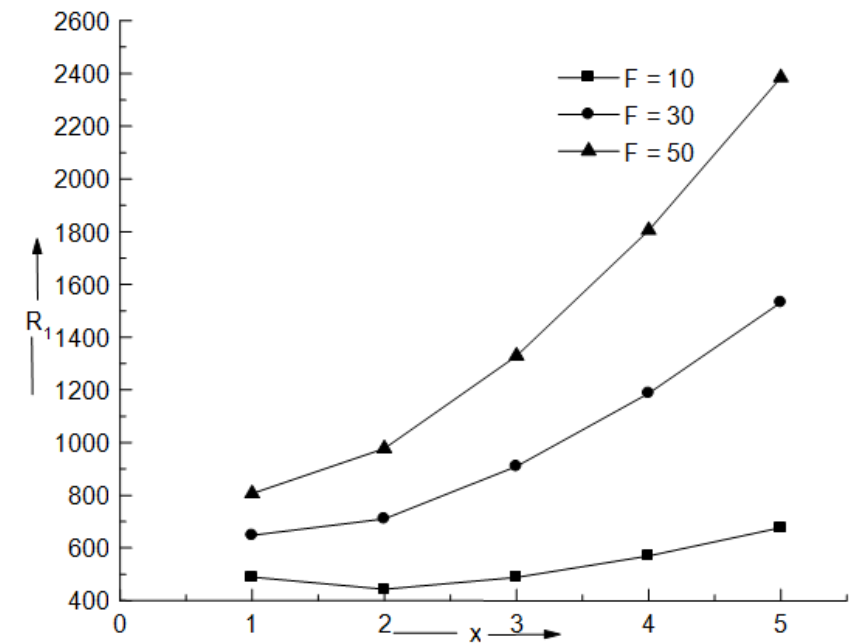

Figure 4: The variation of Rayleigh number $\left(R_{1}\right)$ with wave number $(x)$ for $P=10, Q_{1}=100$

$\mathrm{S}_{1}=10, \varepsilon=0.5$ and $\mathrm{F}=10,30,50$

\section{Some important theorems}

Theorem I: The system is stable or unstable in the presence of the solute gradient, magnetic field and porous medium.

Proof: Multiplying equation (14) by $\mathrm{W}^{*}$, the complex conjugate of $\mathrm{W}$, integrating over the range of $\mathrm{z}$, and making use of equations (15)- (17) together with boundary conditions, we obtain

$$
\begin{aligned}
\left(\frac{\sigma}{\varepsilon}+\frac{1}{P_{l}}\right) I_{1} & +\frac{g \alpha^{\prime} \kappa^{\prime} a^{2}}{v \beta^{\prime}}\left(I_{4}+E^{\prime} q \sigma^{*} I_{5}\right)+\frac{\mu_{e} \varepsilon \eta}{4 \pi \rho_{0} v}\left(I_{6}+p_{2} \sigma^{*} I_{7}\right)+\frac{F}{P_{l}} I_{8} \\
& =\frac{g \alpha \kappa a^{2}}{v \beta}\left(I_{2}+E p_{1} \sigma^{*} I_{3}\right),
\end{aligned}
$$

where

$$
\begin{array}{rlrl}
I_{1} & =\int_{0}^{1}\left(|D W|^{2}+a^{2}|W|^{2}\right) d z, I_{2} & =\int_{0}^{1}\left(|D \Theta|^{2}+a^{2}|\Theta|^{2}\right) d z, \\
I_{3} & =\int_{0}^{1}\left(|\Theta|^{2}\right) d z, & I_{4} & =\int_{0}^{1}\left(|D \Gamma|^{2}+a^{2}|\Gamma|^{2}\right) d z, \\
I_{5} & =\int_{0}^{1}\left(|\Gamma|^{2}\right) d z, & I_{6} & =\int_{0}^{1}\left(\left|D^{2} K\right|^{2}+2 a^{2}|D K|^{2}+a^{4}|K|^{2}\right) d z,
\end{array}
$$




$$
I_{7}=\int_{0}^{1}\left(|D K|^{2}+a^{2}|K|^{2}\right) d z, \quad I_{8}=\int_{0}^{1}\left(\left|D^{2} W\right|^{2}+2 a^{2}|D W|^{2}+a^{4}|W|^{2}\right) d z,
$$

which are all positive definite. Substituting $\sigma=\sigma_{r}+i \sigma_{i}$ and then equating real and imaginary parts of equation (24), we obtain

$$
\begin{aligned}
\sigma_{r}\left(\frac{I_{1}}{\varepsilon}\right. & \left.+\frac{g \alpha^{\prime} \kappa^{\prime} a^{2}}{v \beta^{\prime}} E^{\prime} q I_{5}+\frac{\mu_{e} \varepsilon \eta}{4 \pi \rho_{0} v} p_{2} I_{7}-\frac{g \alpha \kappa a^{2}}{v \beta} E p_{1} I_{3}\right) \\
& =-\left(\frac{1}{P_{l}} I_{1}+\frac{g \alpha^{\prime} \kappa^{\prime} a^{2}}{v \beta^{\prime}} I_{4}+\frac{\mu_{e} \varepsilon \eta}{4 \pi \rho_{0} v} I_{6}+\frac{F}{P_{l}} I_{8}-\frac{g \alpha \kappa a^{2}}{v \beta} I_{2}\right),
\end{aligned}
$$

and

$$
\sigma_{i}\left(\frac{I_{1}}{\varepsilon}-\frac{g \alpha^{\prime} \kappa^{\prime} a^{2}}{v \beta^{\prime}} E^{\prime} q I_{5}-\frac{\mu_{e} \varepsilon \eta}{4 \pi \rho_{0} \nu} p_{2} I_{7}+\frac{g \alpha \kappa a^{2}}{v \beta} E p_{1} I_{3}\right)=0
$$

Equation (26) yields that $\sigma_{r}$ may be positive or negative i.e. there may be stability or instability in the presence of solute gradient, magnetic field and porous medium in couple-stress fluid.

Theorem II: The modes may be oscillatory or non-oscillatory in contrast to the case of no magnetic field and in absence of stable solute gradient.

Proof: Equation (27) yields that $\sigma_{i}=0$ or $\sigma_{i} \neq 0$, which means that the modes may be non-oscillatory or oscillatory. In the absence of stable solute gradient and magnetic field, equation (27) reduces to

$\sigma_{i}\left(\frac{I_{1}}{\varepsilon}+\frac{g \alpha \kappa a^{2}}{v \beta} E p_{1} I_{3}\right)=0$,

and terms in brackets are positive definite. Thus $\sigma_{i}=0$, which means that oscillatory modes are not allowed and the principle of exchange of stabilities is satisfied for a porous medium in couple-stress fluid in the absence of stable solute gradient and magnetic field. This result is true for the porous as well as non-porous medium. The presence of each, the stable solute gradient and the magnetic field brings oscillatory modes (as $\sigma_{i}$ may not be zero) which were non-existent in their absence.

\section{The Overstable case}

Here we discuss the possibility of whether instability may occur as overstability. Put $\frac{\sigma}{\pi^{2}}=i \sigma_{1}$, it being remembered that $\sigma$ may be complex. Since for overstability, we wish to determine the critical Rayleigh number for the onset of instability via a state of pure oscillations, it suffices to find conditions for which (18) will admit of solutions with $\sigma_{1}$ real. Equation (18) becomes

$$
\begin{aligned}
R_{1}=\left(\frac{i \sigma_{1}}{\varepsilon}+\right. & \left.\frac{1}{P}+\frac{\pi^{2} F}{P} \underset{1+x}{-x}\right) \frac{(1+x)\left(1+x+i E p_{1} \sigma_{1}\right)}{x}+S_{1} \frac{\left(1+x+i E p_{1} \sigma_{1}\right)}{\left(1+x+i E^{\prime} q \sigma_{1}\right)} \\
& +\frac{Q_{1}}{\varepsilon} \frac{(1+x)\left(1+x+i E p_{1} \sigma_{1}\right)}{x\left(1+x+i p_{2} \sigma_{1}\right)} .
\end{aligned}
$$

It can be easily shown from equation (29) that $\frac{d R_{1}}{d S_{1}}, \frac{d R_{1}}{d Q_{1}}, \frac{d R_{1}}{d P}$ and $\frac{d R_{1}}{d F}$ yield the same values as given in equations (20)-(23). This means that couple-stress, stable solute gradient and magnetic field have stabilizing effects whereas medium permeability has destabilizing effect on the system for overstable case also. Equating real and imaginary parts of equation (29) and eliminating $R_{1}$ between them, we obtain 


$$
\begin{aligned}
& {\left[\frac{E^{\prime 2} q^{2} p_{2}^{2} \alpha}{\varepsilon}+\frac{E E^{\prime 2} q^{2} p_{1} p_{2}^{2}}{P}\left(1+\pi^{2} F \alpha\right)\right] c_{1}^{2}+} \\
& {[\left(E^{\prime 2} q^{2}+p_{2}^{2}\right)\left\{\alpha^{2}\left(\frac{\alpha}{\varepsilon}+\frac{E p_{1} \overbrace{1+\pi^{2} F \alpha}}{P}\right)\right\}+\frac{E^{\prime 2} q^{2} \alpha Q_{1}\left(E p_{1}-p_{2}\right)}{\varepsilon}+S_{1} \overbrace{\alpha-1} p_{2}^{2}\left(E p_{1}-E^{\prime} q\right)] c_{1}} \\
& +\left[\alpha^{4}\left(\frac{\alpha}{\varepsilon}+\frac{E p_{1} \overbrace{1+\pi^{2} F \alpha}}{P}\right)+S_{1} \alpha^{2}(\alpha-1)\left(E p_{1}-E^{\prime} q\right)+\frac{\alpha^{3}}{\varepsilon} Q_{1}\left(E p_{1}-p_{2}\right)\right]=0,
\end{aligned}
$$

where $1+x=\alpha$ and $\sigma_{1}^{2}=c_{1}$. As $\sigma_{1}$ is real for overstability, the two values of $c_{1}\left(=\sigma_{1}^{2}\right)$ are positive. Equation (30) is quadratic in $c_{1}$ and does not involve any of its roots to be positive if

$$
\left.\begin{array}{ll}
\text { and } & \begin{array}{c}
E p_{1}>p_{2} \\
\text { i.e. }
\end{array} \\
& E p_{1}>E^{\prime} q
\end{array}\right\}
$$

and

$$
\left[\varepsilon+(1-\varepsilon) \frac{\rho_{s} c_{s}^{\prime}}{\rho c^{\prime}}\right] \kappa<\left[\varepsilon+(1-\varepsilon) \frac{\rho_{s} c_{s}}{\rho c}\right] \kappa^{\prime}
$$

where $\rho c, \rho_{s} c_{s}$ and $\rho c^{\prime}, \rho_{s} c_{s}{ }^{\prime}$ denote respectively the heat capacity of fluid, solid matrix and analogous solute capacity of fluid, solid matrix.

Thus, if conditions (32) are satisfied, overstability is impossible and the principle of exchange of stabilities holds good. Therefore, equations (32) are the sufficient conditions for the non-existence of overstability, the violation of which does not necessary involve occurrence of overstability.

In the absence of solute gradient, the sufficient condition for non-existence of overstability reduces to

$$
\kappa<\eta\left[\varepsilon+(1-\varepsilon) \frac{\rho_{s} c_{s}}{\rho c}\right],
$$

which for non-porous medium $(\varepsilon=1)$ further reduces to $\kappa<\eta$ (Chandrasekhar [1981]), for Newtonian fluid], but the introduction of solute gradient introduces an additional sufficient condition

$$
\left[\varepsilon+(1-\varepsilon) \frac{\rho_{s} c_{s}^{\prime}}{\rho c^{\prime}}\right] \kappa<\left[\varepsilon+(1-\varepsilon) \frac{\rho_{s} c_{s}}{\rho c}\right] \kappa^{\prime} .
$$

\section{References}

[1]. D.D. Joseph, Stability of fluid motions (Springer-Verlag Berlin, Vol. I and II., 1976).

[2]. E.R. Lapwood, E.R., Convection of a fluid in a porous medium, Proc. Camb. Phil. Soc., 44, 1948, $508-554$.

[3]. R.A. Wooding., Rayleigh instability of a thermal boundary layer in flow through a porous medium, J. Fluid Mech., 9, 1960, 183192.

[4]. D.A. Nield and A. Bejan, Convection in porous medium (2 $2^{\text {nd }}$ edition) (Springer New York, 1999).

[5]. S. Chandrasekhar, Hydrodynamic and hydromagnetic stability (Dover Publications, New York, 1981)

[6]. G. Veronis, On finite amplitude instability in thermohaline convection, J. Marine Res., 23, 1965, 1-17.

[7]. H. Stommel, A.B. Arons and D. Blanchord., An oceanographical curiosity: the perpetual salt fountain, Deep Sea Res., 3, 1956, 152-153.

[8]. M.E. Stern, The salt fountain and thermohaline convection, Tellus, 12, 1960, 172-175.

[9]. D.A. Nield, The thermohaline convection with linear gradients, J. Fluid Mech., 29, 1967, 545-558.

[10]. H. Tabor and R. Matz, Solar pond project, Solar Energy, 9, 1965, 177-182.

[11]. T.G.L. Shirtcliffe, Lake Bonney Antarctica: Cause of the elevated temperature, J. Geophys. Res., 69, $1964,5257-5268$.

[12]. V.K. Stokes, Couple-stresses in fluids, Phys. Fluids, 9, 1966, 1709-1715. 
[13]. E. Walicki and A. Walicka, Inertia effect in the squeeze film of a couple-stress fluid in biological bearing, Appl. Mech. Engng., 4, 1999, 363-373.

[14]. A.K. Goel., S.C. Agrawal and G.S. Agrawal, Hydromagnetic stability of an unbounded couple stress binary fluid mixture having vertical temperature and concentration gradients with rotation, Ind. J. Pure Appl. Maths., 30, 1999, 991-1001.

[15]. R.C. Sharma, Sunil, Y.D. Sharma and R.S. Chandel, On couple-stress fluid permeated with suspended particles heated from below, Arch. Mech., 54, 2002, 287-298.

[16]. Sunil, R.C. Sharma and M. Pal, On a couple-stress fluid heated from below in a porous medium in the presence of a magnetic field and rotation, J. Porous Media, 5, 2002, 149-158.

[17]. P. Kumar, R. Lal and P. Sharma, Effect of rotation on thermal instability in couple-stress elastico-viscous fluid, $Z$. Fur. Naturforschung A, 59, 2004,407-411. 